\title{
Cuestionario de Esquemas de Young: Adaptación de una versión breve para adolescentes y jóvenes españoles
}

\author{
Esther Calvete \\ Universidad de Deusto, Bilbao, España
}

The Young Schema Questionnaire: Adaptation of a short version to Spanish adolescents and young adults

\begin{abstract}
This study evaluated the psychometric properties of a brief version of the Young-3 Schemas Questionnaire (YSQ-3), which assesses early maladaptive schemas, in Spanish adolescents and young adults. The sample consisted of 1,455 adolescents and young students (54.5\% girls, 15-25 years old). They answered the schemas questionnaire and measures of depression, anxiety, hostility, and non-suicidal self-injury. In addition, a subsample of 842 participants completed the schemas questionnaire again six months later. The results confirm the structure consisting of 18 schemas and support the organization of these schemas in five broader domains. The internal consistency of some subscales was low while satisfactory for the domains. The schemas were stable over time and were significantly associated with several psychological problems. The results allow establishing recommendations for the assessment of maladaptive schemas in future research in the field of clinical psychology.
\end{abstract}

Key words: Early maladaptive schemas; depression; anxiety; hostility; non-suicidal self-injury.

Resumen: Este estudio evaluó las propiedades psicométricas de una versión breve del Cuestionario de Esquemas de Young-3, que evalúa los esquemas desadaptativos tempranos, en adolescentes y jóvenes españoles. La muestra consistió en 1.455 adolescentes y jóvenes estudiantes (54.5\% chicas, 15-25 años). Contestaron el cuestionario de esquemas y medidas de depresión, ansiedad, hostilidad y autolesiones no suicidas. Además, una submuestra de 842 participantes volvió a completar el cuestionario de esquemas breve seis meses más tarde. Los resultados confirman la estructura de 18 esquemas y apoyan la organización de estos en cinco dominios. La consistencia interna de algunas subescalas fue baja mientras que para los dominios fue satisfactoria. Los esquemas fueron estables a lo largo del tiempo y se asociaron significativamente con numerosos problemas psicológicos. Los resultados permiten establecer recomendaciones para la evaluación de los esquemas desadaptativos en la investigación futura en el ámbito de la psicología clínica.

Palabras clave: Esquemas desadaptativos tempranos; depresión, ansiedad; hostilidad; autolesiones no suicidas.

La terapia de esquemas (Young, Klosko y Weishaar, 2003) es un modelo integrador de psicoterapia que ha experimentado un gran auge en los últimos años. Combina elementos cognitivo-conductuales, centrados en la emoción, de vínculo y psicodinámicos, todo ello apoyado en un modelo teórico sobre el desarrollo de los pro-

Recibido: 27 de marzo 2020; aceptado: 14 de abril 2020

Correspondencia: Esther Calvete, Universidad de Deusto, Avenida de las Universidades, 24, 48007-Bilbao, España, Email: esther.calvete@ deusto.es

Agradecimientos: Este estudio fue financiado por el Gobierno Vasco (Ref. IT982-16). blemas psicológicos. Inicialmente fue diseñada para tratar dificultades emocionales de larga duración, tales como los trastornos de personalidad. Sin embargo, a lo largo de los años el uso de la terapia de esquemas se ha extendido a otros numerosos problemas psicológicos, tales como psicosis (Bortolon, Capdevielle, Boulenger, Gely-Nargeot y Raffard, 2013), trastornos de alimentación (Simpson, Morrow, van Vreeswijk y Reid, 2010), depresión (Renner, Arntz, Peeters, Lobbestael y Huibers, 2016) y trastornos de ansiedad (Hawke y Provencher, 2011). Asimismo, la terapia de esquemas ha ido evolucionando en formato de manera que puede aplicarse individualmente, en pareja y en grupo. 
El concepto clave en torno al cual gira la terapia de esquemas es el de esquemas desadaptativos tempranos (early maladaptive schemas, EMS). Los EMS se han descrito como patrones amplios y profundos que incluyen recuerdos, emociones, cogniciones y sensaciones corporales (Young et al., 2003). Esto les diferencia de otros constructos como los esquemas propuestos en el modelo de la terapia cognitiva (Beck, Freeman y Davis, 2012), que son de naturaleza fundamentalmente cognitiva. En cuanto al contenido de los EMS, estos se refieren a la visión del sí mismo y de las relaciones con las demás personas. El modelo de la terapia de esquemas propone que los EMS se desarrollan durante la infancia y adolescencia, y se elaboran a lo largo de la vida. En su origen está implicada la falta de satisfacción de necesidades básicas del niño, como la necesidad de establecer un vínculo seguro con los demás, la necesidad de libertad para expresar emociones, la necesidad de autonomía y competencia y la necesidad de tener unos límites realistas y el auto-control necesarios (Young et al., 2003). Esta concepción teórica que subraya la importancia de la frustración en la satisfacción de necesidades básicas del niño ha dado lugar a numerosos estudios en los que se evalúa el papel que las adversidades tempranas, sobre todo en el seno de la familia, tienen en la predicción de EMS (Calvete, 2014).

Una vez desarrollados los EMS, estos pueden permanecer latentes hasta que son activados por sucesos del ambiente relevantes y al activarse generan emociones intensas y problemas de conducta (Young et al., 2003). Así, por ejemplo, una persona con el esquema de abandono podría experimentar especial ansiedad y angustia ante la perspectiva de la separación de un ser querido. Una persona con el esquema de necesidad de aceptación podría experimentar intenso malestar cuando es objeto de una crítica por parte de otra persona. De esta manera, los EMS actuarían como vulnerabilidades para numerosos problemas psicológicos. Por ello, la investigación actual ha encontrado que los EMS se asocian a problemas psicológicos tales como depresión (Calvete, 2014; Calvete, Orue y Hankin, 2013), trastornos de alimentación (Pauwels et al., 2013), autolesiones suicidas y no suicidas (Flink et al., 2017; Khosravani, Sharifi Bastan, Samimi Ardestani y Jamaati Ardakani, 2017), consumo de sustancias (Khosravani, Alvani, Seidisarouei, Amirinezhad y Shojaee, 2017; Lewis, Lumley y Grunberg, 2015), trastornos de personalidad (Frías et al., 2018), entre otros. Además, los EMS se han mostrado estables a lo largo del tiempo (Calvete et al., 2013).

En este contexto, la evaluación de los EMS es una tarea central. Young y colaboradores desarrollaron el Young Schema Questionnaire (YSQ) para evaluar los principales EMS implicados en los problemas emocionales. La primera versión consistía en 205 ítems que evaluaban 16 EMS (Schmidt, Joiner, Young y Telch, 1995). Estos estaban agrupados en cinco grandes dominios, en función de la necesidad básica del niño no satisfecha que estaba implicada en el origen del esquema. Los cinco dominios son: (1). Desconexión y rechazo, que incluye esquemas consistentes en la expectativa de que las demás personas no estarán disponibles para proporcionar la seguridad, afecto y respecto necesarios de una forma estable, (2) autonomía y ejecución dañados, que incluye esquemas con contenidos relacionados con la falta de capacidad para funcionar o ejecutar conductas de una forma autónoma y exitosa, (3) Limites dañados, que incluye esquemas que evidencian déficits en los límites internos y en la responsabilidad hacia otras personas, (4) Foco en los demás, que se caracteriza por un foco excesivo en los deseos de otras personas y en agradar a estas, con un coste para la satisfacción de las necesidades propias, y (5) Sobrevigilancia e inhibición, que incluye esquemas relacionados con un énfasis excesivo en el control de los sentimientos y conductas propias con el fin de evitar errores y lograr altos estándares de conducta. Aunque la investigación sobre el modelo EMS de Young et al. (2003) ha sugerido que los EMS bajo el dominio de Desconexión y Rechazo son los más perjudiciales, y muchos EMS pueden estar presentes en personas con cuadros clínicos muy diversos, el uso del YSQ ha permitido establecer cierta especificidad en cuanto a los EMS que pueden ser más relevante para cada problema psicopatológico. Por ejemplo, los esquemas del dominio de desconexión y rechazo han mostrado ser especialmente relevantes para problemas como la depresión (Calvete, Orue y Hankin, 2013; Yiğit, K1lıç, Guzey Yiğit y Çelik, 2018), algunos esquemas del dominio de foco en los demás y de autonomía dañada se asocian con la ansiedad social (Calvete y Orue, 2008) y los esquemas de abuso (dentro del dominio de desconexión y rechazo) y grandiosidad (dominio de limites dañados) son relevantes para la conducta agresiva (Tremblay y Dozois, 2009). Recientemente, se ha dirigido la atención sobre el rol de los EMS en las conductas de autolesiones no suicidas, que son altamente prevalentes en adolescentes y jóvenes (Calvete, Orue, Aizpuru y Brotherton, 2015). En un estudio se encontró que los jóvenes que se autolesionaban puntuaban más alto en aislamiento social, inhibición emocional y más bajo en grandiosidad (Lewis et al., 2015). En otro estudio, se encontró que los jóvenes con más autolesiones puntuaban más alto en la mayoría de los EMS (Arthurs y Tan, 2017).

El YSQ no solo se ha utilizado en investigación, sino que constituye una herramienta importante en la terapia 
de esquemas. Los pacientes contestan el cuestionario con el fin de facilitar los EMS de mayor relevancia clínica e informar así la selección de estrategias terapéuticas (Young et al., 2003).

Dado que la versión inicial resultaba muy larga tanto en el ámbito clínico como en el de investigación, pronto se desarrolló una versión más corta: el YSQ-Short Form, YSQ-SF, Young y Brown, 1994), que consistía en 75 ítems y evaluaba 15 EMS. Esta versión se convirtió en el cuestionario más popular para evaluar los EMS, siendo adaptado a numerosos idiomas, incluyendo el español (Calvete, Estévez, De Arroyabe y Ruiz, 2005). En 2006, surgió una nueva versión en la que se añadían tres nuevos esquemas, pasando así a evaluar 18 EMS mediante 90 ítems (YSQ-3, Young, 2006). Al igual que ocurrió con su predecesora, la nueva versión fue adaptada a numerosos idiomas, incluyendo el español (Calvete, Orue y González-Diez, 2013) y sus propiedades psicométricas fueron evaluadas en numerosos estudios (Bach, Simonsen, Christoffersen y Kriston, 2017; Bouvard, Denis y Roulin, 2018). En general, estos estudios han confirmado la existencia de los 18 esquemas, habiendo más discrepancias en lo referente a las dimensiones de segundo orden (i.e., dominios) que agrupan estos esquemas (Calvete, Orue y González-Diez, 2013; Sakulsriprasert, Phukao, Kanjanawong y Meemon, 2016).

Aunque el YSQ-3 es considerablemente más corto que el original YSQ, aún puede resultar muy largo, especialmente cuando se utiliza con adolescentes y jóvenes en estudios donde estos deben completar numerosas medidas. Por este motivo, recientemente, se ha desarrollado una versión corta del YSQ-3 (Santos, Vagos y Rijo, 2018), que fue denominada the Brief form of the Young Schema Questionnaire for Adolescents (B-YSQ-A). Los autores seleccionaron tres ítems de cada subescala, basándose en criterios estadísticos y de análisis del contenido de los ítems. Así, se eligieron ítems caracterizados por las mayores correlaciones media entre-ítems, cargas factoriales e impacto en la consistencia interna de la subescala a la que pertenecían. Tres expertos en terapia de esquemas evaluaron el contenido de los ítems para garantizar que los ítems excluidos no contenían aspectos esenciales del esquema.

De esta manera, la versión breve consiste en solo 54 ítems y acorta sustancialmente el tiempo de aplicación. Los autores pudieron confirmar la estructura de 18 subescalas con el B-YSQ-A y en general obtuvieron coeficientes de consistencia interna adecuados, salvo para los esquemas de grandiosidad y de sacrificio (Santos et al., 2018). Aunque los resultados también apoyaron que los 18 esquemas pueden organizarse en los cinco dominios propuestos por Young, el modelo jerárquico no fue el que mostró el mejor ajuste. En un trabajo posterior, se mostró que el modelo de medida del B-YSQ-A era invariante en muestra portuguesa y brasileña (Borges, Vagos, Dell'Aglio y Rijo, 2020). Los coeficientes de consistencia interna fueron en general adecuados salvo unas pocas excepciones (p.ej., el esquema de grandiosidad y de sacrificio).

La revisión anterior muestra que la evaluación de los EMS es de gran relevancia en el ámbito de la psicología clínica. Los EMS constituyen un importante factor de vulnerabilidad para numerosos problemas psicológicos y su evaluación es necesaria no solo con fines de investigación sino también en el ámbito de la intervención psicológica. Por ello, la posibilidad de disponer de una versión breve en español es de gran interés en ambos ámbitos. El objetivo de este estudio es extender el trabajo realizado con el B-YSQ-A (Santos et al., 2018) y evaluar la versión breve en español del YSQ-3 (YSQ-3-B) dirigida a adolescentes y jóvenes. Con el fin de homogeneizar versiones, se optó por seleccionar los mismos ítems utilizados en la versión portuguesa, que como se ha indicado se basaron en criterios tanto estadísticos como de contenido. Crear versiones similares en cuanto a composición de ítems es importante porque permite comparar los resultados obtenidos en diferentes países y avanzar mejor en el conocimiento sobre la terapia de esquemas. Como parte del objetivo planteado, en este estudio se evaluó la estructura factorial del cuestionario breve, probando los dos modelos con más apoyo en la literatura previa: un modelo consistente en los 18 factores y un modelo jerárquico en el que cinco grandes dominios explican la variabilidad entre los 18 factores. Además, se evaluó la estabilidad de las puntuaciones del cuestionario a lo largo del tiempo y se estudiaron las correlaciones entre las puntuaciones obtenidas, tanto con la versión larga como con la corta, con cuatro tipos de problemas psicológicos (ansiedad, depresión, autolesiones y hostilidad).

\section{Método}

\section{Participantes}

La muestra fue de conveniencia y consistió en 1455 adolescentes y jóvenes estudiantes (54.5\% chicas) de 11 centros educativos de Bizkaia, incluyendo centros de educación secundaria obligatoria y estudios universitarios. Las edades oscilaron entre 15 y 25 años (edad media $=19.96, D E=2.61$ ). Según la información de los participantes sobre educación e ingresos de los padres, los niveles socioeconómicos fueron: $13.4 \%$ bajo, $19.2 \%$ medio bajo, $22.6 \%$ medio, $16.7 \%$ medio alto y $28.1 \%$ 
alto. Se realizó un seguimiento al de seis meses en aproximadamente la mitad de las aulas, participando un total de 852 adolescentes y jóvenes de la muestra original $(58.56 \%)$. No hubo diferencias en ninguna de las variables del estudio entre los adolescentes que completaron el seguimiento y los que no.

\section{Medidas}

Cuestionario de Esquemas de Young-3 (YSQ-3; Young, 2006), versión española de Calvete, Orue y González-Diez, 2013 a partir de la traducción de Cid y Torrubia, 2010). El YSQ-3 tiene 90 ítems que evalúan los 18 EMS propuestos por Young et al. (2003). Los EMS se agrupan en cinco dominios. El dominio de desconexión y rechazo incluye los esquemas de abandono (p.ej., Me siento aferrado/a las personas a las que estoy muy unido/a porque tengo miedo de que me abandonen), abuso (p.ej., Presiento que la gente se aprovechará de mí), privación emocional (p.ej., En general, no he tenido a mi lado personas que me dieran cariño, apoyo y afecto), imperfección (p.ej., Nadie que yo desee, querría estar cerca de mí, si me conociese realmente) y aislamiento social (p.ej., Me siento poco integrado en los grupos). El dominio de autonomía y ejecución dañados incluye los esquemas de dependencia (p.ej., No me siento capaz de arreglármelas por mí mismo en las cosas de cada día), vulnerabilidad al daño o enfermedad (p.ej., No puedo escapar a la sensación de que algo malo va a ocurrir), apego (p.ej., A menudo siento que no tengo una identidad independiente de la de mis padres o de mi pareja) y fracaso (p.ej., La mayoría de las personas están más capacitadas que yo en temas de estudios y de rendimiento.). El dominio de limites dañados incluye los esquemas de grandiosidad (p.ej., Odio que me limiten o que no me dejen hacer lo que quiera) y autocontrol insuficiente (p.ej., No consigo obligarme a hacer aquello que no me gusta, aunque sepa que es por mi bien). El dominio de foco en los demás incluye los esquemas de sacrificio (p.ej., Estoy tan ocupado haciendo cosas por las personas que me importan, que me queda poco tiempo para mí mismo), subyugación (p.ej., Siento que no tengo otra opción que ceder ante los deseos de los demás, de lo contrario se vengarán de mí o me rechazarán de alguna manera) y necesidad de aceptación (p.ej., Los elogios son más valiosos para mí si los demás se dan cuenta). Por último, el dominio de sobrevigilancia e inhibición incluye los esquemas de pesimismo (p.ej., Por mucho cuidado que tenga, casi siempre algo irá mal), inhibición emocional (p.ej., La gente me ve como alguien emocionalmente rígido), castigo (p.ej., Si cometo un error, merezco ser castigado) y altos estándares (p.ej., Siento una presión constante por hacer las cosas y darlas por terminadas). Cada escala se evalúa con 5 ítems con un formato de respuesta de 6 puntos que oscila entre 1 (completamente falso) y 6 (me describe perfectamente). En el estudio en el primer tiempo se aplicó la versión larga con el fin de obtener indicadores tanto de la versión larga como de la breve.

Symptoms Check List-90-R (SCL-90-R; Derogatis, 2004)). La versión española del SCL-90-R ha mostrado buenas propiedades psicométricas en estudiantes españoles (Caparrós-Caparrós, Villar-Hoz, Juan-Ferrer y Viñas-Poch, 2007). Aunque el SCL-90-R mide numerosos problemas psicológicos, en este estudio únicamente se utilizaron los 40 ítems incluidos en las escalas de ansiedad generalizada, depresión y hostilidad. Los ítems se responden mediante una escala de cinco puntos con un rango entre 1 ( nada) y 5 (mucho). En este estudio los coeficientes alpha fueron $.86, .89$ y .86 , respectivamente, para ansiedad, depresión y hostilidad.

La Escala de Evaluación Funcional de la Autolesión (The Functional Assessment of Self-Mutilation Scale, FASM; Lloyd, Kelley y Hope, 1997; versión en español de Calvete et al., 2015). Se utilizó el FASM para evaluar autolesiones no suicidas durante los últimos 12 meses. En la primera parte del FASM, los adolescentes indicaron con qué frecuencia se habían involucrado en 10 formas diferentes de autolesiones, incluyendo cortarse, hurgarse una herida, auto-golpearse, rasparse la piel hasta sangrar y morderse a sí mismo, entra otras. En la segunda parte del FASM, aquellos adolescentes que informaron haber realizado autolesiones indicaron las funciones de estas. Se les preguntó con qué frecuencia se habían involucrado en autolesiones con fines de autoreforzamiento automático (p.ej., por sentir algo, por aliviar la tensión) y de refuerzo social (p.ej., por obtener atención, evitar ir al colegio, etc.) mediante 22 ítems que debían responderse con un rango de 0 (nunca) a 3 (a menudo). Se obtuvieron puntuaciones para las funciones de reforzamiento. Los coeficientes alfa fueron .70 y .85 para refuerzo automático y social, respectivamente.

\section{Procedimiento}

Los estudiantes universitarios $(n=621)$ fueron contactados e invitados a participar en las aulas. En el caso de estudiantes de educación obligatoria secundaria ( $n=$ 834), la invitación se realizó en primer lugar a través de los responsables de los centros e incluyó el envío de consentimiento informado pasivo a los progenitores o representantes legales de los menores. Los participantes completaron las medidas en las aulas y el proceso llevó 
aproximadamente media hora. A los participantes se les invitó a indicar un código o clave, conocido solo por ellos, con el fin de realizar un seguimiento seis meses más tarde.

\section{Análisis Estadísticos}

Los análisis factoriales confirmatorios se realizaron mediante el programa LISREL-10. Dado que las variables se distribuían con normalidad se utilizó el método de máxima verosimilitud. En consistencia con la investigación previa, se probaron los dos modelos con mayor evidencia: Un modelo consistente en 18 factores correlacionados y un modelo jerárquico en el que cinco dominios explicaban los 18 factores de primero orden. Ambos modelos se probaron tanto para la versión larga $(90$ ítems) como para la breve (54 ítems). Con el fin de evaluar la adecuación de los modelos se utilizaron la raíz cuadrada media de error de aproximación (RMSEA), el índice de ajuste comparativo (CFI), el índice de ajuste no normativo (NNFI) y raíz cuadrada media residual (SRMR) para evaluar la bondad de ajuste del modelo. Los valores del CFI y NNFI mayores que .90, valores del RMSEA menores que .06 y valores de SRMR menores que .08 reflejan un buen ajuste (Hu y Bentler, 1999). Como se ha indicado, para la versión breve se utilizaron los mismos ítems que en la versión breve portuguesa. Los coeficientes de correlación y estadísticos descriptivos se obtuvieron mediante el programa IBM-SPSS-26.

\section{Resultados}

\section{Estructura factorial del YSQ-3-B}

En cuanto a la versión larga del YSQ-3, el modelo de 18 factores correlacionados mostró un ajuste excelente, $\chi^{2}(3762, n=1455)=15870$, RMSEA $=.047(.046 ; .048)$, $\mathrm{NNFI}=.96, \mathrm{CFI}=.96, \mathrm{SRMR}=.057$. El modelo jerárquico mostró indicadores de ajuste ligeramente peores pero también satisfactorios, $\chi^{2}(3887, n=1455)=21137$, RMSEA $=.055(.055 ; .056)$, NNFI $=.95$, CFI $=.95$, $\mathrm{SRMR}=.071$. El cambio en $\chi^{2}$ fue estadísticamente significativo, $\Delta \chi^{2}(125)=5267, p<.001$. Tres ítems presentaron cargas factoriales por debajo del punto de corte de .40 (ítem 28 en dependencia, ítem 65 en apego e ítem 90 en castigo).

A continuación, se probó el modelo de medida para la versión breve (YSQ-3-B). En este caso, el modelo de 18 factores correlacionados mostró también un ajuste excelente, $\chi^{2}(1224, n=1455)=4183$, RMSEA $=.041(.039$; $.042), \mathrm{NNFI}=.96, \mathrm{CFI}=.97, \mathrm{SRMR}=.042 . \mathrm{El}$ modelo jerárquico mostro índices adecuados, si bien algo infe- riores, $\chi^{2}(1349, n=1455)=7482$, RMSEA $=.056(.055$; $.057), \mathrm{NNFI}=.95, \mathrm{CFI}=.95, \mathrm{SRMR}=.065 . \mathrm{El}$ cambio en $\chi^{2}$ fue estadísticamente significativo, $\Delta \chi^{2}(125)=3299$, $p<.001$. Todas las cargas factoriales fueron superiores a .40 excepto la carga factorial de segundo orden de altos estándares en el dominio de sobrevigilancia e inhibición que fue .25 , si bien fue estadísticamente significativa.

Finalmente, se probaron los modelos de medida para la versión corta en el seguimiento $(N=842)$ y los resultados fueron similares: $\chi^{2}(1224, n=842)=3102$, RM$\mathrm{SEA}=.043(.041 ; .045), \mathrm{NNFI}=.97, \mathrm{CFI}=.97$, SRMR $=.045$ para el modelo de primer orden y $\chi^{2}(1349, n=$ $842)=4709$, RMSEA $=.054(.053 ; .056), \mathrm{NNFI}=.96$, $\mathrm{CFI}=.96, \mathrm{SRMR}=.063$ para el jerárquico. El cambio en $\chi^{2}$ fue estadísticamente significativo, $\Delta \chi^{2}(125)=1410$, $p<.001$. La Tabla 1 muestra las cargas factoriales del YSQ-3-B en los dos tiempos.

\section{Consistencia interna de las subescalas y consistencia temporal}

La Tabla 2 recoge los coeficientes alfa de Cronbach de las subescalas de esquemas y las escalas de dominios. Tal y como se observa, en la versión corta del YSQ-3 varias subescalas no alcanzan el valor de .70 (p.ej., vulnerabilidad, apego, subyugación, sacrificio, grandiosidad y autocontrol insuficiente) y lo mismo sucede con algunas subescalas de la versión larga (p.ej., dependencia, apego, grandiosidad). En cambio, las escalas de dominios, en general, presentan valores de consistencia interna adecuados, con la excepción de Límites dañados, que queda un poco por debajo del valor de .70. La Tabla 2 también muestra las medias y desviaciones típicas de todas las puntuaciones del YSQ-3 y del YSQ-3-B.

\section{Correlaciones entre las puntuaciones del YSQ-3 y sintomas psicológicos}

Finalmente se obtuvieron los coeficientes de correlación entre las puntuaciones obtenidas mediante la versión larga y breve del YSQ-3 y los síntomas de depresión, ansiedad, hostilidad y autolesiones no suicidas. En el caso de estas últimas, un total de 334 participantes indicaron haberlas realizado y contestaron la escala de razones. Los resultados se muestran en la Tabla 3, donde se observan numerosas correlaciones estadísticamente significativas. En general el patrón de correlaciones obtenido entre esquemas y síntomas psicológicos es muy similar para el YSQ-3 y para el YSQ-3-B. Además, en la mayoría de los casos los mayores coeficientes se obtienen para los dominios de esquemas más que para esque- 
Tabla 1. Cargas factoriales de los ítems en el YSQ-3-B

\begin{tabular}{|c|c|c|c|c|c|}
\hline & Tiempo 1 & Tiempo 2 & & Tiempo 1 & Tiempo 2 \\
\hline Privación & & & Subyugación & & \\
\hline Item 36 & 0.71 & 0.67 & Item 21 & 0.67 & 0.65 \\
\hline Item 50 & 0.84 & 0.82 & Item 38 & 0.41 & 0.40 \\
\hline Item 57 & 0.58 & 0.60 & Item 44 & 0.67 & 0.67 \\
\hline Abandono & & & Sacrificio & & \\
\hline Item 41 & 0.73 & 0.74 & Item 26 & 0.66 & 0.62 \\
\hline Item 48 & 0.74 & 0.74 & Item 34 & 0.60 & 0.55 \\
\hline Item 70 & 0.77 & 0.78 & Item 54 & 0.58 & 0.74 \\
\hline Abuso & & & Necesidad de aceptación & & \\
\hline Item 4 & 0.58 & 0.67 & Item 79 & 0.58 & 0.64 \\
\hline Item 14 & 0.68 & 0.71 & Item 82 & 0.74 & 0.75 \\
\hline Item 58 & 0.72 & 0.77 & Item 88 & 0.55 & 0.64 \\
\hline Imperfección & & & Grandiosidad & & \\
\hline Item 2 & 0.67 & 0.75 & Item 24 & 0.50 & 0.53 \\
\hline Item 8 & 0.71 & 0.74 & Item 40 & 0.51 & 0.55 \\
\hline Item 64 & 0.50 & 0.52 & Item 67 & 0.48 & 0.49 \\
\hline Aislamiento & & & Autocontrol insuficiente & & \\
\hline Item 3 & 0.57 & 0.68 & Item 13 & 0.46 & 0.52 \\
\hline Item 43 & 0.70 & 0.66 & Item 72 & 0.64 & 0.67 \\
\hline Item 74 & 0.80 & 0.77 & Item 75 & 0.61 & 0.62 \\
\hline Fracaso & & & Inhibición & & \\
\hline Item 9 & 0.75 & 0.77 & Item 52 & 0.73 & 0.74 \\
\hline Item 45 & 0.76 & 0.79 & Item 46 & 0.57 & 0.52 \\
\hline Item 51 & 0.83 & 0.82 & Item 62 & 0.76 & 0.76 \\
\hline Dependencia & & & Altos estándares & & \\
\hline Item 25 & 0.40 & 0.44 & Item 23 & 0.66 & 0.80 \\
\hline Item 16 & 0.52 & 0.49 & Item 11 & 0.61 & 0.68 \\
\hline Item 59 & 0.69 & 0.78 & Item 39 & 0.43 & 0.45 \\
\hline Vulnerabilidad & & & Pesimismo & & \\
\hline Item 6 & 0.51 & 0.60 & Item 77 & 0.78 & 0.78 \\
\hline Item 53 & 0.52 & 0.49 & Item 80 & 0.77 & 0.76 \\
\hline Item 68 & 0.57 & 0.60 & Item 83 & 0.79 & 0.82 \\
\hline Apego & & & Castigo & & \\
\hline Item 19 & 0.53 & 0.54 & Item 78 & 0.77 & 0.77 \\
\hline Item 60 & 0.47 & 0.53 & Item 84 & 0.58 & 0.61 \\
\hline Item 65 & 0.40 & 0.44 & Item 87 & 0.78 & 0.79 \\
\hline
\end{tabular}

mas específicos. La excepción es la asociación entre el esquema de pesimismo y la mayoría de los problemas psicológicos, que tiende a ser mayor que la obtenida con la puntuación en el dominio de sobrevigilancia e inhibi- ción en el que se encuadra este esquema. También destacan las altas correlaciones entre los dominios de desconexión y rechazo y autonomía dañada con los síntomas de depresión. 
Tabla 2. Coeficientes alfa y correlaciones entre puntuaciones en el tiempo 1 y 2.

\begin{tabular}{|c|c|c|c|c|c|c|}
\hline & \multicolumn{2}{|c|}{$\begin{array}{c}\text { T1 } \\
\text { YSQ-3 }\end{array}$} & \multicolumn{2}{|c|}{$\begin{array}{c}\text { T1 } \\
\text { YSQ-3-B }\end{array}$} & \multirow{2}{*}{$\begin{array}{c}\text { T2 } \\
\text { YSQ-3-B } \\
\text { Alfa }\end{array}$} & \multirow{2}{*}{$\begin{array}{c}\text { T1-T2 } \\
\text { YSQ-3-B } \\
\text { Correlación test-retest* }^{*}\end{array}$} \\
\hline & $M(D T)$ & Alfa & $M(D T)$ & Alfa & & \\
\hline Desconexión y rechazo & $53.96(17.30)$ & .90 & $35.09(11.00)$ & .83 & .86 & .71 \\
\hline Privación emocional & $9.28(4.93)$ & .83 & $5.63(3.17)$ & .74 & .73 & .63 \\
\hline Abandono & $14.50(5.74)$ & .80 & $9.67(4.04)$ & .78 & .80 & .59 \\
\hline Abuso/desconfianza & $11.82(4.55)$ & .75 & $7.77(3.23)$ & .69 & .76 & .63 \\
\hline Imperfección & $8.20(3.53)$ & .73 & $5.17(2.42)$ & .62 & .68 & .60 \\
\hline Aislamiento social & $10.17(4.23)$ & .75 & $6.85(2.99)$ & .64 & .65 & .68 \\
\hline Autonomía dañada & $41.72(12.75)$ & .84 & $26.01(9.22)$ & .82 & .83 & .65 \\
\hline Fracaso & $9.95(4.47)$ & .85 & $6.07(3.05)$ & .82 & .83 & .60 \\
\hline Dependencia & $9.76(3.46)$ & .52 & $4.56(2.23)$ & .74 & .75 & .51 \\
\hline Vulnerabilidad & $12.14(4.86)$ & .68 & $7.69(3.35)$ & .55 & .58 & .61 \\
\hline Apego & $9.88(3.77)$ & .59 & $6.27(2.59)$ & .46 & .50 & .49 \\
\hline Foco en los demás & $42.34(10.15)$ & .80 & $25.96(6.71)$ & .73 & .76 & .63 \\
\hline Subyugación & $10.62(3.98)$ & .68 & $6.75(2.76)$ & .58 & .57 & .58 \\
\hline Autosacrificio & $16.20(4.63)$ & .75 & $9.73(2.95)$ & .64 & .67 & .56 \\
\hline Necesidad de aceptación & $15.52(5.09)$ & .75 & $9.48(3.40)$ & .67 & .73 & .59 \\
\hline Limites dañados & $28.19(7.68)$ & .74 & $16.12(4.84)$ & .74 & .69 & .64 \\
\hline Grandiosidad & $14.51(4.42)$ & .62 & $7.30(3.55)$ & .49 & .52 & .53 \\
\hline Autocontrol insuficiente & $13.68(4.80)$ & .70 & $8.22(3.10)$ & .60 & .62 & .57 \\
\hline Sobrevigilancia e inhibición & $11.63(5.43)$ & .82 & $24.57(7.01)$ & .70 & .74 & .65 \\
\hline Inhibición emocional & $42.09(11.24)$ & .81 & $7.90(2.93)$ & .72 & .71 & .66 \\
\hline Estándares altos & $17.08(4.44)$ & .61 & $9.35(3.20)$ & .57 & .65 & .56 \\
\hline Pesimismo & $13.13(5.55)$ & .80 & $7.31(3.79)$ & .81 & .83 & .67 \\
\hline Castigo & $11.88(4.46)$ & .71 & $7.92(3.40)$ & .75 & .76 & .56 \\
\hline
\end{tabular}

Nota. ${ }^{*}$ Todas las correlaciones entre puntuaciones del tiempo 1 y 2 son estadísticamente significativas con $p<.001$.

\section{Discusión}

El interés por el estudio de los EMS ha crecido de manera exponencial en los últimos años (Taylor, Bee y Haddock, 2017; Yakın, Gençöz, Steenbergen y Arntz, 2019). Los EMS son el concepto clave dentro de la terapia de esquemas y, de acuerdo con el modelo conceptual de esta terapia, desempeñan un papel central en el origen y mantenimiento de numerosos problemas psicológicos. Por ello, es de gran importancia disponer de medidas adecuadas para su evaluación. El presente estudio tuvo como objetivo evaluar la versión breve del YSQ-3 (i.e., el YSQ-3-B), la última versión disponible del cuestionario de esquemas, en adolescentes y jóvenes españoles. Tomando como referencia la versión portuguesa (Santos et al., 2018), se procedió al análisis de las propiedades psicométricas principales de esta versión.

Los resultados confirman la estructura del YSQ-3-B consistente en 18 factores en correspondencia con los 18 EMS tanto en la versión larga como breve. Aunque los indicadores de ajuste fueron algo peores, los resultados también indican que una versión jerárquica en la que los cinco dominios explican las puntuaciones en los 18 esquemas es aceptable. Estos resultados son consistentes con la mayoría de los obtenidos en estudios previos, que confirman la existencia de los 18 factores de primer orden pero que indican resultados mixtos para los dominios o factores de segundo orden (Calvete, Orue y González-Diez, 2013; Santos et al., 2018). No obstante, dado que el modelo jerárquico sigue obteniendo indicadores de ajuste aceptables, es recomendable su uso. Los domi- 
Tabla 3. Coeficientes de Correlación entre las puntuaciones del YSQ-3 y Síntomas Psicológicos

\begin{tabular}{|c|c|c|c|c|c|c|c|c|c|c|}
\hline & \multicolumn{2}{|c|}{ Depresión } & \multicolumn{2}{|c|}{ Ansiedad } & \multicolumn{2}{|c|}{ Hostilidad } & \multicolumn{2}{|c|}{ ARA } & \multicolumn{2}{|c|}{ ARS } \\
\hline & larga & breve & larga & breve & larga & breve & larga & breve & larga & breve \\
\hline Desconexión y rechazo & $.59 * *$ & $.56^{* *}$ & $.32 * *$ & $.32 * *$ & $.37 * *$ & $.38^{* *}$ & $.32 * *$ & $.32 * *$ & $.18^{* *}$ & $.17 *$ \\
\hline Privación emocional & $.42^{* *}$ & $.38^{* *}$ & $.24^{* *}$ & $.22 * *$ & $.26^{* *}$ & $.25^{* *}$ & $.30^{* *}$ & $.29^{* *}$ & $.17^{*}$ & $.17^{*}$ \\
\hline Abandono & $.47 * *$ & $.40^{* *}$ & $.28^{* *}$ & $.23^{* *}$ & $.29 * *$ & $.25^{* *}$ & $.18^{*}$ & $.11^{*}$ & $.15^{*}$ & .09 \\
\hline Abuso/desconfianza & $.46^{* *}$ & $.40 * *$ & $.26^{* *}$ & $.26^{* *}$ & $.35^{* *}$ & $.35^{* *}$ & $.24 * *$ & $.26^{* *}$ & $.15^{*}$ & $.18^{* *}$ \\
\hline Imperfección & $.41^{* *}$ & $.37 * *$ & $.18^{* *}$ & $.16^{*}$ & $.26^{* *}$ & $.21^{* *}$ & $.20^{* *}$ & $.18^{* *}$ & .07 & .02 \\
\hline Aislamiento social & $.43^{* *}$ & $.38^{* *}$ & $.21^{* *}$ & $.22 * *$ & $.22 * *$ & $.23^{* *}$ & $.27^{* *}$ & $.27^{* *}$ & $.11^{*}$ & $.13^{*}$ \\
\hline Autonomía dañada & $.51^{* *}$ & $.41^{* *}$ & $.38^{* *}$ & $.33^{* *}$ & $.38^{* *}$ & $.33^{* *}$ & $.22 *$ & $.18^{* *}$ & $.21^{*}$ & $.17^{* *}$ \\
\hline Fracaso & $.37 * *$ & $.34 * *$ & $.26^{* *}$ & $.24 * *$ & $.31 * *$ & $.26^{* *}$ & $.13^{*}$ & $.13^{*}$ & $.11^{*}$ & .10 \\
\hline Dependencia & $.38^{* *}$ & $.32 * *$ & $.25^{* *}$ & $.24^{* *}$ & $.27^{* *}$ & $.24 * *$ & $.12 *$ & $.11^{*}$ & $.11^{*}$ & .08 \\
\hline Vulnerabilidad & $.40^{* *}$ & $.30 * *$ & $.30 * *$ & $.24 * *$ & $.30 * *$ & $.26^{* *}$ & $.18^{* *}$ & $.15^{*}$ & $.15^{*}$ & $.16^{*}$ \\
\hline Apego & $.38^{* *}$ & $.33^{* *}$ & $.31^{* *}$ & $.20^{* *}$ & $.26^{* *}$ & $.20^{* *}$ & $.25^{* *}$ & $.16^{*}$ & $.28^{* *}$ & $.21^{* *}$ \\
\hline Foco en los demás & $.48^{* *}$ & $.45^{* *}$ & $.25^{* *}$ & $.22 * *$ & $.31^{* *}$ & $.27^{* *}$ & $.17^{*}$ & $.16^{*}$ & $.17^{*}$ & $.16^{*}$ \\
\hline Subyugación & $.46^{* *}$ & $.42^{* *}$ & $.20^{* *}$ & $.16^{*}$ & $.28 * *$ & $.21^{* *}$ & $.20^{* *}$ & $.15^{*}$ & $.13^{*}$ & $.14^{*}$ \\
\hline Autosacrificio & $.35^{* *}$ & $.30^{* *}$ & $.12^{*}$ & .10 & $.15^{*}$ & $.15 *$ & .09 & .09 & .04 & .05 \\
\hline Necesidad de aceptación & $.28^{* *}$ & $.28^{* *}$ & $.23 * *$ & $.22 * *$ & $.25 * *$ & $.23 * *$ & .10 & $.11^{*}$ & $.19^{* *}$ & $.16^{*}$ \\
\hline Limites dañados & $.39^{* *}$ & $.34 * *$ & $.37 * *$ & $.35^{* *}$ & $.40 * *$ & $.36^{* *}$ & $.25^{*}$ & $.28 * *$ & $.17^{*}$ & $.19 * *$ \\
\hline Grandiosidad & $.29^{* *}$ & $.24^{* *}$ & $.35^{* *}$ & $.26^{* *}$ & $.31^{* *}$ & $.26^{* *}$ & $.27^{* *}$ & $.26^{* *}$ & $.23^{* *}$ & $.21 * *$ \\
\hline Autocontrol insuficiente & $.36^{* *}$ & $.30 * *$ & $.27^{* *}$ & $.29^{* *}$ & $.36^{* *}$ & $.32 * *$ & $.16^{*}$ & $.19^{* *}$ & .06 & .09 \\
\hline Sobrevigilancia e inhibición & $.47^{* *}$ & $.33^{* *}$ & $.29 * *$ & $.21^{* *}$ & $.30^{* *}$ & $.21^{* *}$ & $.27^{* *}$ & $.28^{* *}$ & $.22^{* *}$ & $.19^{* *}$ \\
\hline Inhibición emocional & $.27^{* *}$ & $.26^{* *}$ & .08 & .05 & $.19^{* *}$ & $.18^{* *}$ & .09 & .09 & .02 & .02 \\
\hline Estándares altos & $.19^{* *}$ & .11 & .10 & .02 & .04 & .02 & $.14 *$ & .10 & .04 & .04 \\
\hline Pesimismo & $.51^{* *}$ & $.51^{* *}$ & $.34^{* *}$ & $.36^{* *}$ & $.36^{* *}$ & $.37^{* *}$ & $.28 * *$ & $.31^{* *}$ & $.24 * *$ & $.23 * *$ \\
\hline Castigo & $.35 * *$ & $.32 * *$ & $.21^{* *}$ & $.17^{* *}$ & $.25^{* *}$ & $.23 * *$ & $.19 * *$ & .10 & $.20^{* *}$ & $.12 *$ \\
\hline
\end{tabular}

Nota . ARA $=$ Autolesiones por reforzamiento automático, ARS $=$ Autolesiones por reforzamiento social. Las correlaciones con ARA y ARS se obtuvieron en la submuestra de 334 participantes que reportaron autolesiones no suicidas.

$* p<.05, * * p<.001$.

nios sintetizan la información de numerosos esquemas y el disponer de unas pocas puntuaciones globales puede ser de gran utilidad en la investigación clínica, por ejemplo, cuando se trata de probar modelos predictivos entre variables. La inclusión de 18 esquemas individuales en dichos modelos puede ser inviable desde un punto de vista estadístico.

En cuanto a la consistencia interna de las subescalas, al igual que sucede en otros estudios anteriores (Calvete, Orue y González-Diez, 2013), los coeficientes alpha para algunos esquemas son inadecuados, especialmente para la versión breve en la que cada esquema es evaluado únicamente con tres ítems. En concreto, la consistencia interna fue especialmente baja para los esquemas de apego y vulnerabilidad (dominio de autonomía dañada), imperfección y aislamiento social (dominio de desconexión y rechazo), subyugación y autosacrificio (dominio de foco en los demás), grandiosidad y bajo autocontrol (dominio de límites dañados) y altos estándares (dominio de sobrevigilancia e inhibición). En cambio, los dominios de esquemas mostraron en general buenos coeficientes de consistencia interna, con la excepción del dominio de limites dañados, para el cual en el tiempo 2 fue ligeramente inferior al límite recomendado de .70 . Estos resultados en cuanto a consistencia interna son también consistentes con la recomendación del uso de puntuaciones de dominios en investigación. Esto no quiere decir que no deban utilizarse puntuaciones de esquemas específicos en algunas situaciones ya que algunos de ellos pueden ser particularmente relevantes para 
algunos problemas psicológicos tal y como se discute más adelante.

Otro aspecto reseñable es la alta estabilidad de los esquemas a lo largo de los seis meses de seguimiento. De modo consistente con la teoría (Young et al., 2003), los esquemas tienden a perdurar ya que actuarían como rasgos de personalidad. Esta teoría establece que a través de diversos mecanismos como el mantenimiento de esquemas a través del foco de la atención en las circunstancias que confirman los esquemas o la evitación de situaciones activadoras, los EMS se muestran resistentes al cambio y contribuyen al desarrollo y persistencia de los problemas psicológicos. Diversos estudios apoyan esta concepción sobre la estabilidad de los EMS a lo largo del tiempo y su papel predictivo de problemas emocionales y conductuales (Calvete, Orue y Hankin, 2013). Más aún, en el presente estudio la estabilidad fue especialmente alta para el dominio de desconexión y rechazo (.71), lo cual es concordante con la importancia dada a los esquemas de este dominio en el modelo de terapia de esquemas. Este dominio incluye algunos esquemas que se consideran incondicionados y nucleares (Young et al., 2003) y que de un modo dinámico pueden influir en el funcionamiento de otros esquemas.

Por último, el estudio arroja datos sobre la asociación de los EMS y los dominios de esquemas con numerosos problemas psicológicos. Los resultados muestran que los EMS están asociados de manera extensa con la mayoría de los problemas psicológicos evaluados en el estudio, destacando probablemente el papel del dominio de desconexión y rechazo. Como se ha mencionado, este dominio se caracterizaría por esquemas incondicionados y nucleares, los cuales se hipotetizan como particularmente relevantes para el malestar de las personas (Young et al., 2003). El patrón de asociaciones es muy similar para la versión larga y breve del YSQ-3. Los resultados también muestran que, en general, los dominios están más prominentemente asociados con los problemas psicológicos que los esquemas específicos. Una excepción es el dominio de sobrevigilancia e inhibición, en el que varios esquemas son poco relevantes para los problemas psicológicos evaluados en el estudio, pero en el que los esquemas de castigo y sobre todo de pesimismo están claramente asociados con los problemas psicológicos. Es posible que los esquemas de inhibición emocional y altos estándares sean relevantes para otros problemas psicológicos no incluidos en este estudio, como algunos trastornos de personalidad (p.ej., trastorno de personalidad esquizoide, trastorno de personalidad obsesivo-compulsiva).

Mientras que numerosos estudios previos han examinado las asociaciones entre EMS y síntomas de ansiedad y depresión (Borges et al., 2020; Yiğit et al., 2018) e in- cluso hostilidad y agresividad (Temblay y Dozois, 2009), el presente estudio arroja información sobre el área menos evaluada de la relación entre EMS y autolesiones no suicidas. En general y en consistencia con otros estudios (Arthurs y Tan, 2017), las funciones de las autolesiones se asociaron a numerosos EMS, destacando su asociación con los dominios de desconexión y rechazo y de limites dañados así como con el esquema de pesimismo. Es importante además que la asociación fue más evidente con la función de reforzamiento automático de las autolesiones, la cual está implicada en procesos de desregulación emocional (Nock y Prinstein, 2004). Esto sugeriría que los EMS podrían asociarse con las autolesiones no suicidas a través de la potenciación de mecanismos disfuncionales de regulación emocional, lo cual es consistente con la asociación entre EMS y desregulación emocional y rumiación (Alba y Calvete, 2019a; Yakın et al., 2019)

Este estudio presenta limitaciones que ofrecen oportunidades para la investigación futura. La primera limitación se refiere a que está basado en una muestra de la comunidad. Si bien los esquemas están presentes en la población en general (Young et al., 2003), sería conveniente examinar las propiedades psicométricas del YSQ3-B en población clínica. Es posible que algunas de las subescalas que en la muestra de este estudio mostraron coeficientes alpha inadecuados muestren un mejor funcionamiento en población clínica, donde los esquemas pueden estar especialmente activados. Más aún, es posible que las asociaciones entre esquemas y síntomas psicológicos se evidencien en mayor medida en población clínica. Por ejemplo, un estudio reciente mostró que varios dominios de esquemas se asociaban más intensamente a la depresión en población clínica que comunitaria (Alba y Calvete, 2019b). Una segunda limitación es el uso exclusivo de autoinformes, que puede contribuir a incrementar la asociación entre variables. En este sentido, sería interesante incluir informes diagnósticos en futuros estudios. La tercera limitación es que este estudio solo incluyó cuatro tipos de problemas psicológicos y sería interesante evaluar las asociaciones del YSQ-3-B con otras problemáticas como trastornos de personalidad. Finalmente, sería conveniente evaluar el rol mediador del cambio en esquemas en los efectos de la terapia de esquemas en problemas psicológicos. Según una reciente revisión, la terapia de esquemas reduce los EMS pero hay una falta de estudios de mediación rigurosos (Taylor et al., 2017)

A pesar de las limitaciones, este estudio contribuye con datos sobre una versión breve del YSQ-3. Aunque algunas subescalas presentan consistencia interna baja debido a que se utilizan solamente tres ítems por subes- 
cala, en conjunto el cuestionario presenta buenas propiedades psicométricas. De los resultados se deriva la recomendación de utilizar las puntuaciones de dominios de esquemas, las cuales presentan buena consistencia interna y además se asocian más intensamente con los problemas psicológicos. Esto no implica que algunos esquemas específicos puedan ser relevantes y puedan utilizarse en el estudio de determinados problemas psicológicos.

\section{Referencias}

Alba, J. y Calvete, E. (2019a). Bidirectional relationships between stress, depressive symptoms, and cognitive vulnerabilities in adolescents. Journal of Social and Clinical Psychology. https://doi.org/10.1521/jscp.2019.38.2.87

Alba, J. y Calvete, E. (2019b). Relaciones entre depresión, estrés $\mathrm{y}$ esquemas disfuncionales tempranos en adolescentes. Behavioral Psychology/ Psicologia Conductual.

Arthurs, S. D. y Tan, J. C. H. (2017). Personality Traits, Early Maladaptive Schemas, and Severity of Nonsuicidal SelfInjury. Psi Chi Journal of Psychological Research, 22, 181192. https://doi.org/10.24839/2325-7342.jn22.3.181

Bach, B., Simonsen, E., Christoffersen, P. y Kriston, L. (2017). The young schema questionnaire 3 short form (YSQ-S3): Psychometric properties and association with personality disorders in a Danish mixed sample. European Journal of Psychological Assessment, 33, 134-143. https://doi. org/10.1027/1015-5759/a000272

Beck, A. T., Freeman, A. y Davis, D. D. (2012). Cognitive therapy for personality disorders. En The Oxford handbook of personality disorders. Oxford University Press.

Borges, J. L., Vagos, P., Dell'Aglio, D. D. y Rijo, D. (2020). Crosscultural Validation of the Young Schema Questionnaire for Adolescents in Portuguese and Brazilian Samples. International Journal of Cognitive Therapy. https://doi. org/10.1007/s41811-020-00067-6

Bortolon, C., Capdevielle, D., Boulenger, J. P., Gely-Nargeot, M. C. y Raffard, S. (2013). Early maladaptive schemas predict positive symptomatology in schizophrenia: A cross-sectional study. Psychiatry Research, 209, 361-366. https://doi. org/10.1016/j.psychres.2013.03.018

Bouvard, M., Denis, A. y Roulin, J. L. (2018). Psychometric Properties of the French Version of the Young Schema Questionnaire - Short Form 3 (YSQ-S3). Spanish Journal of Psychology, 3, 1-14. https://doi.org/10.1017/sjp.2018.66

Calvete, E. (2014). Emotional abuse as a predictor of early maladaptive schemas in adolescents: Contributions to the development of depressive and social anxiety symptoms. Child Abuse and Neglect, 38, 735-746. https://doi. org/10.1016/j.chiabu.2013.10.014

Calvete, E., Estévez, A., De Arroyabe, E. L. y Ruiz, P. (2005). The schema questionnaire -short form: Structure and relationship with automatic thoughts and symptoms of affective disorders. European Journal of Psychological Assessment. https://doi. org/10.1027/1015-5759.21.2.90

Calvete, E., y Orue, I. (2008). Ansiedad social y esquemas cognitivos disfuncionales. Psicologia Conductual, 16, 5-21.
Calvete, E., Orue, I., Aizpuru, L. y Brotherton, H. (2015). Prevalence and functions of non-suicidal self-injury in Spanish adolescents/Prevalencia y funciones de autolesiones no suicidas en adolescentes españoles. Psicothema, 27, 223229. https://doi.org/10.7334/psicothema2014.262

Calvete, E., Orue, I. y González-Diez, Z. (2013). An examination of the structure and stability of early maladaptive schemas by means of the Young Schema Questionnaire-3. European Journal of Psychological Assessment, 29, 283-290.

Calvete, E., Orue, I. y Hankin, B. L. (2013). Transactional relationships among cognitive vulnerabilities, stressors, and depressive symptoms in adolescence. Journal of abnormal child psychology, 41, 399-410.

Caparrós-Caparrós, B., Villar-Hoz, E., Juan-Ferrer, J. y ViñasPoch, F. (2007). Symptom Check-List-90-R: Fiabilidad, datos normativos y estructura factorial en estudiantes universitarios. International Journal of Clinical and Health Psychology, 7, 781-794.

Cid, J. y Torrubia, R. (2010). Spanish version of the Young Schemas Questionnaire-3 (manuscrito no publicado). Mental Health Management Area, Girona

Derogatis, L. R. (2004). SCL-90-R. En Encyclopedia of psychology, Vol. 7. https://doi.org/10.1037/10522-079

Esmaeilian, N. (s. f.). Early maladaptive schemas and borderline personality disorder features in a non-clinical sample: $A$ network analysis, 1-41. https://doi.org/10.1002/cpp.2360

Flink, N., Lehto, S. M., Koivumaa-Honkanen, H., Viinamäki, H., Ruusunen, A., Valkonen-Korhonen, M. y Honkalampi, K. (2017). Early maladaptive schemas and suicidal ideation in depressed patients. European Journal of Psychiatry, 31, 8792. https://doi.org/10.1016/j.ejpsy.2017.07.001

Frías, Á., Navarro, S., Palma, C., Farriols, N., Aliaga, F., Salvador, A., ... Solves, L. (2018). Early maladaptive schemas associated with dimensional and categorical psychopathology in patients with borderline personality disorder. Clinical Psychology and Psychotherapy, 25, e30-e41. https://doi. org/10.1002/cpp.2123

Hawke, L. D. y Provencher, M. D. (2011). Schema Theory and Schema Therapy in Mood and Anxiety Disorders: A review. Journal of Cognitive Psychotherapy, 25, 257-276. https://doi. org/10.1891/0889-8391.25.4.257

Hu, L. T. y Bentler, P. M. (1999). Cutoff criteria for fit indexes in covariance structure analysis: Conventional criteria versus new alternatives. Structural Equation Modeling. https://doi. org/10.1080/10705519909540118

Khosravani, V., Alvani, A., Seidisarouei, M., Amirinezhad, A. y Shojaee, D. (2017). Early maladaptive schemas, behavioral inhibition/approach systems, and defense styles in the users of natural and synthetic substances and healthy subjects. Journal of Substance Use, 22, 168-175. https://doi.org/10.3109/14659 891.2016.1166272

Khosravani, V., Sharifi Bastan, F., Samimi Ardestani, M. y Jamaati Ardakani, R. (2017). Early maladaptive schemas and suicidal risk in an Iranian sample of patients with obsessive-compulsive disorder. Psychiatry Research, 255, 441-448. https://doi. org/10.1016/j.psychres.2017.06.080

Lewis, S. P., Lumley, M. N. y Grunberg, P. H. (2015). Early maladaptive schemas and non-suicidal self-injury among young adults: A preliminary investigation. Counselling 
Psychology Quarterly, 28, 386-402. https://doi.org/10.1080/0 9515070.2015.1074887

Lloyd, E. E., Kelley, M. L. y Hope, T. (1997, April). Self-mutilation in a community sample of adolescents: Descriptive characteristics and provisional prevalence rates. En Annual meeting of the Society for Behavioral Medicine, New Orleans, LA, USA.

Nock, M. K. y Prinstein, M. J. (2004). A functional approach to the assessment of self-mutilative behavior. Journal of Consulting and Clinical Psychology. https://doi.org/10.1037/0022-006X.72.5.885

Pauwels, E., Claes, L., Smits, D., Dierckx, E., Muehlenkamp, J. J., Peuskens, H. y Vandereycken, W. (2013). Validation and reliability of the young schema questionnaire in a flemish inpatient eating disorder and alcohol and substance use disorder sample. Cognitive Therapy and Research, 37, 647656. https://doi.org/10.1007/s10608-012-9501-4

Renner, F., Arntz, A., Peeters, F. P. M. L., Lobbestael, J. y Huibers, M. J. H. (2016). Schema therapy for chronic depression: Results of a multiple single case series. Journal of Behavior Therapy and Experimental Psychiatry, 51, 66-73. https://doi. org/10.1016/j.jbtep.2015.12.001

Sakulsriprasert, C., Phukao, D., Kanjanawong, S. y Meemon, N. (2016). The reliability and factor structure of Thai Young Schema Questionnaire-Short Form 3. Asian Journal of Psychiatry, 24, 85-90. https://doi.org/10.1016/j.ajp.2016.09.011

Santos, L., Vagos, P. y Rijo, D. (2018). Dimensionality and Measurement Invariance of a Brief Form of the Young Schema Questionnaire for Adolescents. Journal of Child and Family Studies, 27, 2100-2111. https://doi.org/10.1007/s10826-0181050-3

Simpson, S. G., Morrow, E., van Vreeswijk, M. y Reid, C. (2010). Group schema therapy for eating disorders: A pilot study.
Frontiers in Psychology, 1, 1-10. https://doi.org/10.3389/ fpsyg.2010.00182

Schmidt, N. B., Joiner, T. E., Young, J. y Telch, M.J. (1995). The Schema Questionnaire: Investigation of psychometric properties and the hierarchical structure of a measure of maladaptive schemas. Cognitive Therapy and Research, 19, 295-321.

Taylor, C. D. J., Bee, P. y Haddock, G. (2017). Does schema therapy change schemas and symptoms? A systematic review across mental health disorders. Psychology and Psychotherapy: Theory, Research and Practice, 90, 456-479. https://doi. org/10.1111/papt.12112

Tremblay, P. F. y Dozois, D. J. (2009). Another perspective on trait aggressiveness: Overlap with early maladaptive schemas. Personality and Individual Differences, 46, 569-574.

Yakın, D., Gençöz, T., Steenbergen, L. y Arntz, A. (2019). An integrative perspective on the interplay between early maladaptive schemas and mental health: The role of selfcompassion and emotion regulation. Journal of Clinical Psychology, 75, 1098-1113. https://doi.org/10.1002/jclp. 22755

Yiğit, İ., Kılıç, H., Guzey Yiğit, M. y Çelik, C. (2018). Emotional and physical maltreatment, early maladaptive schemas, and internalizing disorders in adolescents: a multi-group path model of clinical and non-clinical samples. Current Psychology. https://doi.org/10.1007/s12144-018-0068-4

Young, J. \& Brown, G. (1994). Young Schemas Questionnaire S1. New York, NY: Cognitive Therapy Center

Young, J., Klosko, J.S. y Weishaar, M. E. (2003). Schema therapy. A practitioner's guide. Guilford Press.

Young, J. (2006). Young Schema Questionnaire-3. New York, NY: Cognitive Therapy Center. 
